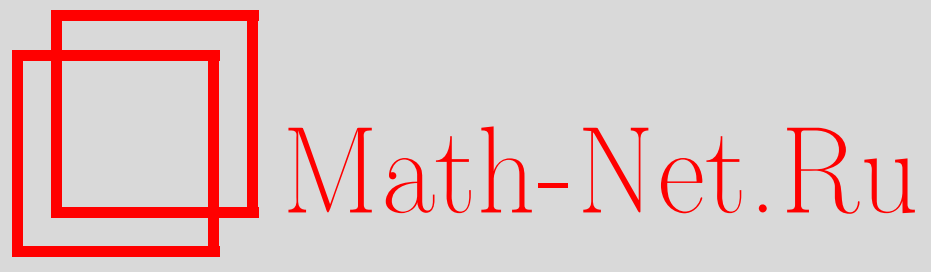

И. С. Тюрин, Уточнение верхних оценок констант в теореме Ляпунова, УМH, 2010, том 65, выпуск 3, 201-202

DOI: https://doi.org/10.4213/rm9337

Использование Общероссийского математического портала Math-Net.Ru подразумевает, что вы прочитали и согласны с пользовательским соглашением http://www . mathnet.ru/rus/agreement

Параметры загрузки:

IP: 54.198 .67 .100

26 апреля 2023 г., $14: 25: 21$

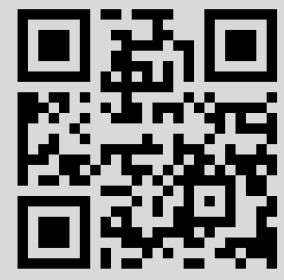




\section{Уточнение верхних оценок констант в теореме Ляпунова}

\section{И. С. Тюрин}

Пусть независимые случайные величины (с. в.) $X_{1}, \ldots, X_{n}$ невырождены, центрированы и обладают конечными третьими абсолютными моментами $\beta_{1}, \ldots, \beta_{n}$. Обозначим их дисперсии $\sigma_{1}^{2}, \ldots, \sigma_{n}^{2}$ и положим $\sigma^{2}(n):=\sigma_{1}^{2}+\cdots+\sigma_{n}^{2}$. Величина $\varepsilon_{n}:=$ $\left(\beta_{1}+\cdots+\beta_{n}\right) / \sigma^{3}(n)$, называемая дробью Ляпунова, позволяет эффективно оценивать расстояние в метрике Колмогорова между нормированной суммой $S_{n}:=\left(X_{1}+\right.$ $\left.\cdots+X_{n}\right) / \sigma(n)$ и стандартной нормальной с. в. $N$. Существует такая наименьшая постоянная $C$, что для каждого $n \in \mathbb{N}$ и произвольных с. в. $X_{1}, \ldots, X_{n}$ справедливо неравенство

$$
\rho\left(S_{n}, N\right):=\sup _{x \in \mathbb{R}}\left|\mathrm{P}\left(S_{n} \leqslant x\right)-\mathrm{P}(N \leqslant x)\right| \leqslant C \varepsilon_{n} .
$$

Точное значение константы $C$ не известно до сих пор, но существуют ее верхние и нижние оценки. Более полувека назад Эссеен [1] показал, что $C \geqslant 0.409 \ldots$. Верхние оценки последовательно улучшались на протяжении последних 67 лет, наиболее точная на данный момент была получена автором [2] в 2009 г. Удалось установить, что $C \leqslant 0.6379$, а в частном случае независимых одинаково распределенных (н. о. р.) слагаемых $C \leqslant 0.5894$. В настоящей работе в развитие результатов [2] доказана

Теорема. Неравенство (1) имеет место с константой $C \leqslant 0.5606$, а в случае одинаково распределенных слагаемых $C \leqslant 0.4785$.

Нам потребуются несколько вспомогательных утверждений. При $\gamma, t \in \mathbb{R}$ положим

$$
b(t, \gamma):=\left\{\begin{array}{lll}
-t^{2}+2 \gamma a|t|^{3}, & \text { если } & \gamma|t|<M \\
-2 \gamma^{-2}(1-\cos \gamma t), & \text { если } & M \leqslant \gamma|t| \leqslant 2 \pi \\
0, & \text { если } & \gamma|t|>2 \pi .
\end{array}\right.
$$

Здесь $a:=\max _{x>0}\left\{\left(\cos x-1+x^{2} / 2\right) / x^{3}\right\}, M$ - соответствующая точка максимума. Пусть $f_{n}(t):=\mathrm{E} e^{i t S_{n}}, \varphi(t):=e^{-t^{2} / 2}, \delta_{n}(t):=\left|f_{n}(t)-\varphi(t)\right|, t \in \mathbb{R}$.

Лемма 1 [2]. Для любого $t \in \mathbb{R}$ имеем $\delta_{n}(t) \leqslant \min \left\{h_{1}\left(\varepsilon_{n},|t|\right), h_{2}\left(\varepsilon_{n},|t|\right)\right\}$, где

$$
\begin{gathered}
h_{1}(\varepsilon, t):=\varepsilon \varphi(t) \int_{0}^{t} \frac{s^{2}}{2} \exp \left(\frac{s^{2}}{2}\right) d s, \\
h_{2}(\varepsilon, t):=\varepsilon \varphi(t)\left(\int_{0}^{\min \{t, A\}} \frac{s^{2}}{2} \exp \left(\frac{s^{2} \varepsilon^{2 / 3}}{2}\right) d s+\int_{A}^{\max \{t, A\}} \frac{s^{2}}{2 l} \exp \left(2 a \varepsilon s^{3}\right) d s\right) .
\end{gathered}
$$

Здесь $A:=\varepsilon^{-1 / 3} /(6 a), l:=\inf _{t \geqslant 0}\left\{\exp \left(-t^{2} / 2+2 a t^{3}\right)\right\} \approx 0.624489$.

Лемма 2 [2]. Если $X_{j}, j \geqslant 1,-$ н.о.р. с.в., то при всех $t \in \mathbb{R} u n \in \mathbb{N}$ выполнено $\delta_{n}(t) \leqslant h_{3}\left(\varepsilon_{n}, n,|t|\right)$, а при $n \geqslant m u t \in \mathbb{R}$ имеем $\delta_{n}(t) \leqslant h_{4}\left(\varepsilon_{n}, m,|t|\right)$, где

$$
\begin{aligned}
h_{3}(\varepsilon, n, t) & :=\varepsilon \varphi(t) \int_{0}^{t}\left(1+\frac{b\left(s, \varepsilon+n^{-1 / 2}\right)}{n}\right)^{\frac{n-1}{2}} \frac{s^{2}}{2} \exp \left(\frac{s^{2}}{2}\right) d s, \\
h_{4}(\varepsilon, m, t) & :=\varepsilon \varphi(t) \int_{0}^{t} \exp \left(\frac{m-1}{2 m} b\left(s, \varepsilon+m^{-1 / 2}\right)+\frac{s^{2}}{2}\right) \frac{s^{2}}{2} d s .
\end{aligned}
$$

Кроме того, из результатов работы [3] следует, что верна

Лемма 3. Для всех $t \in \mathbb{R} u n \in \mathbb{N}$ верно $\left|f_{n}(t)\right|^{2} \leqslant h_{5}^{2}\left(\varepsilon_{n}, t\right):=\exp \left(b\left(t, 2 \varepsilon_{n}\right)\right)$. А в случае н.о.р. величин справедливы также следующие оченки:

$$
\begin{array}{lll}
\left|f_{n}(t)\right|^{2} \leqslant h_{6}^{2}\left(\varepsilon_{n}, n, t\right):=\left(1+n^{-1} b\left(t, \varepsilon_{n}+n^{-1 / 2}\right)\right)^{n}, & t \in \mathbb{R}, & n \in \mathbb{N}, \\
\left|f_{n}(t)\right|^{2} \leqslant h_{7}^{2}\left(\varepsilon_{n}, m, t\right):=\exp \left(b\left(t, \varepsilon_{n}+m^{-1 / 2}\right)\right), & t \in \mathbb{R}, & n \geqslant m .
\end{array}
$$


Обозначим $D(\varepsilon, n)$ наименьшую величину такую, что для любого набора $n$ с. в. $X_{1}, \ldots, X_{n}$ с $\varepsilon_{n}=\varepsilon$ выполнено $\rho\left(S_{n}, N\right) \leqslant D(\varepsilon, n) \varepsilon_{n}$. Положим $D(\varepsilon):=\sup _{n \geqslant 1} D(\varepsilon, n)$. Тогда $C=\sup _{\varepsilon>0} D(\varepsilon)$ и достаточно показать, что $D(\varepsilon, n)$ при всех возможных значениях $\varepsilon$ и $n$ не превосходит 0.5606 (а в случае н. о. р. с. в. 0.4785 ). Для $\varepsilon \geqslant 1 / 0.5606$ (соответственно $\varepsilon \geqslant 1 / 0.4785)$ последнее очевидно, поскольку $\rho\left(S_{n}, N\right) \leqslant 1$. Кроме того, обозначим $\hat{\sigma}^{2}(n):=\sigma^{2}(n)-\max \left\{\sigma_{1}^{2}, \ldots, \sigma_{n}^{2}\right\}$ и положим $\hat{\varepsilon}_{n}:=\left(\beta_{1}+\cdots+\beta_{n}\right) / \hat{\sigma}^{3}(n)$, $\varepsilon_{n}^{(k)}:=\left(\sigma_{1}^{k}+\cdots+\sigma_{n}^{k}\right) / \hat{\sigma}^{k}(n), k=3,4$. Тогда, согласно [4; (I.52)], при $\hat{\varepsilon}_{n}+\varepsilon_{n}^{(3)} \leqslant 0.2$ имеем

$$
\rho\left(S_{n}, N\right) \leqslant 0.27283 \hat{\varepsilon}_{n}+0.19948 \varepsilon_{n}^{(3)}+0.09116 \varepsilon_{n}^{(4)}+0.00095\left(\hat{\varepsilon}_{n}+\varepsilon_{n}^{(3)}\right)^{2} .
$$

В частности, из этого неравенства вытекает, что $D(\varepsilon) \leqslant 0.5606$ при $\varepsilon \leqslant 0.02$ (а в случае н. о. p. с.в. $D(\varepsilon) \leqslant 0.4785$ при $\varepsilon \leqslant 0.037)$. Следовательно, можно ограничиться рассмотрением $\varepsilon \in I_{1}=[0.02 ; 1 / 0.5606]$, а в случае н.о.р. величин $-\varepsilon \in I_{2}=$ $[0.037 ; 1 / 0.4785]$. Доказательство теоремы для этих значений $\varepsilon$ основано на неравенстве Правитца [4]

$$
\begin{aligned}
\frac{\rho\left(S_{n}, N\right)}{\varepsilon_{n}} \leqslant & \frac{1}{\varepsilon_{n}} \\
& \left(\int_{-U_{0}}^{U_{0}} \frac{1}{U}\left|K\left(\frac{u}{U}\right)\right| \cdot\left|\delta_{n}(u)\right| d u+\int_{U_{0}<|u| \leqslant U} \frac{1}{U}\left|K\left(\frac{u}{U}\right)\right| \cdot\left|f_{n}(u)\right| d u\right. \\
& \left.+\int_{-U_{0}}^{U_{0}}\left|\frac{1}{U} K\left(\frac{u}{U}\right)-\frac{i}{2 \pi u}\right| \cdot|\varphi(u)| d u+\int_{|u|>U_{0}}\left|\frac{\varphi(u)}{2 \pi u}\right| d u\right)
\end{aligned}
$$

где $K(u):=(1-|u|) / 2+i\left((1-|u|) \cot (\pi u)+\pi^{-1} \operatorname{sgn}(u)\right) / 2,0<U_{0} \leqslant U$.

Из $(3)$ вытекает, что $D(\varepsilon)$ не превосходит величины $D^{*}\left(\varepsilon, U_{0}, U\right)$, которая получается из правой части $(3)$, если величины $\left|f_{n}(t)\right|,\left|\delta_{n}(t)\right|$ заменить их оценками $h_{5}(\varepsilon, t)$, $\min \left\{h_{1}(\varepsilon, t), h_{2}(\varepsilon, t)\right\}$, и подобрать параметры $U_{0}, U$ так, чтобы полученное выражение оказалось как можно меньше. Описанная процедура была проведена для 460 значений $\varepsilon$, рассредоточенных по отрезку $I_{1}$. Чтобы получить оценки для промежуточных точек, было использовано то, что $\varepsilon D^{*}\left(\varepsilon, U_{0}, U\right)$ не убывает по $\varepsilon$.

Экстремальное значение величины $D^{*}\left(\varepsilon, U_{0}, U\right)=0.56054$ возникло при $\varepsilon=0.5092$, $U_{0}=2.4852, U=5.9508$.

В случае н. о. р. с. в. при фиксированном $\varepsilon$ выбиралось $m$ - некоторое натуральное число и для значений $n<m$ давались индивидуальные оценки $D(\varepsilon, n)$. В правую часть (3) вместо $\left|\delta_{n}(t)\right|$ и $\left|f_{n}(t)\right|$ подставлялись их оценки $h_{3}(\varepsilon, n, t)$ и $h_{6}(\varepsilon, n, t)$, после чего применялась описанная выше процедура подбора параметров. Для $n \geqslant m$ величины $D(\varepsilon, n)$ оценивались равномерно. При этом в правой части (3) использовались оценки $h_{4}(\varepsilon, m, t)$ и $h_{7}(\varepsilon, m, t)$. Для н. о. р. с. в. экстремальное значение 0.47849 было достигнуто при $\varepsilon=0.3536, n=8, U_{0}=2.6157, U=8.9115$. Итак, теорема верна во всех рассмотренных случаях.

Автор выражает глубокую признательность профессору А. В. Булинскому за полезные обсуждения и замечания.

\section{Список литературы}

[1] C. G. Esseen, Skand. Aktuarietidskr., 39 (1956), 160-170. [2] И. С. Тюрин, Докл. РАН, 429:3 (2009), 312-316. [3] H. Prawitz, Scand. Actuar. J., 1975, 21-28. [4] H. Prawitz, Scand. Actuar. J., 1975, 145-156.

И. С. Тюрин (I. S. Tyurin)

Московский государственный университет им. М. В. Ломоносова

E-mail: itiurin@gmail.com
Представлено А. В. Булинским Принято редколлегией 15.12.2009 